\title{
Tumours disrupt the immune scenery
}

How does tumour burden affect systemic immune responses? Are there lasting immune effects after tumour clearance? The dynamicity of the immune system during cancer development is still rather poorly understood. Allen, Hiam et al. have used mass cytometry to characterize the immune landscape over time in response to tumour development across five cancer tissues in eight common mouse tumour models. They have demonstrated that tumour growth dynamically alters the systemic immune landscape throughout the body and can be reverted by the surgical removal of the tumour.

The tumour microenvironment (TME) was examined in established but pre-terminal mouse tumour models to reflect the patient population most often treated with immunotherapies, revealing unique immune profiles depending on the various tumour origins (breast, pancreas, colon, skin or brain). Determination of the quantity and activity of different types of peripheral immune cells (sampled from bone marrow, blood, spleen and lymph nodes near the untreated tumours) showed all three breast cancer models exclusively presented with consistent splenic remodelling with an increase in frequencies of neutrophils, monocytes and eosinophils and a reduction in $\mathrm{B}$ and $\mathrm{T}$ cells resulting from tumour burden. The same shift in the immune state was further observed in whole blood samples of untreated patients with breast cancer when compared to matched controls, suggesting a large disruption of the immune macroenvironment and paving the way for potential improvements in the immunotherapy applications in the clinic.

Exploring the dynamics of global immune remodelling during breast tumour growth in mice over 35 days revealed nonlinear progressive changes in the systemic $\mathrm{T}$ cell composition, such as the positive correlation of absolute cell counts of tumour-infiltrating leukocytes with tumour size supporting the anti-tumour immune response with tumour growths. Notably, in the two breast cancer models investigated, the immune profiles within the TME remained distinct from those detected in the peripheral sites. Systemically, the most dramatic changes occurred in the lymph nodes.

To understand the degree of $\mathrm{T}$ cell alteration and immune reorganization, Allen, Hiam et al. investigated the immune organs during tumour growth. Indeed, all $\mathrm{T}$ cell subsets were altered across tissues over time. Particularly, $\mathrm{CD}_{103}{ }^{+}$regulatory $\mathrm{T}$ cells and $\mathrm{CD} 90^{\text {high }} \mathrm{CD} 4^{+} \mathrm{T}$ cells were found to be reduced overall in the tumour but expanded in the lymph nodes with disease progression. $\mathrm{CD} 8^{+}$ $\mathrm{T}$ cells increased in the tumour but not the spleen, with the majority of cells expressing programmed cell death protein 1 (PD1) and CD69, strongly suggesting T cell dysfunction and alteration between the TME and periphery. Interestingly, PD1 and PD1 ligand 1 (PDL1) were dynamically expressed in the periphery with PDL1 mostly on non-classical monocytes and classical dendritic cells and PD1 on eosinophils, neutrophils and T cells, suggesting the possibility of inconsistent sensitivity to checkpoint blockade over time.

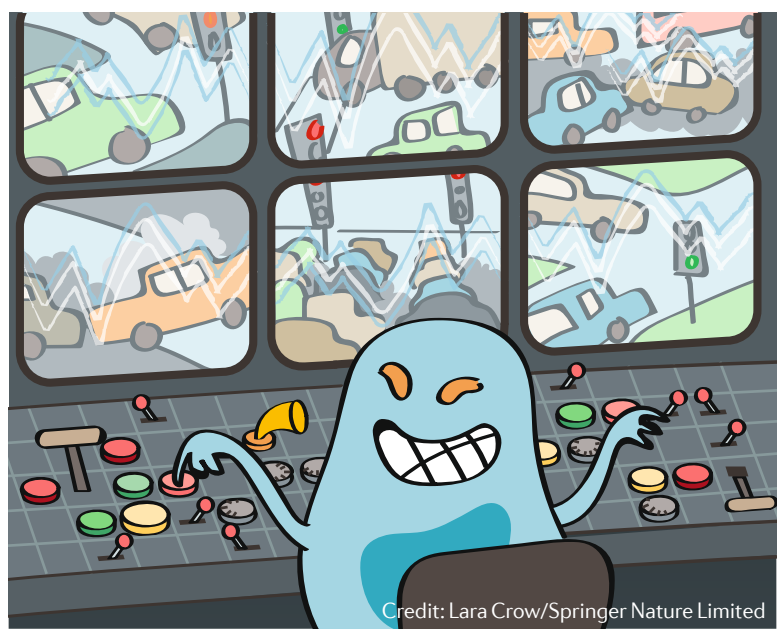

All mice with cancers presented with weakened immune responses to both bacterial and viral infection. While the pathogens were cleared from the spleen, the overall cellular response was altered, and cell extrinsic mechanisms were found to supress systemic T cell function in tumour-bearing mice. These outcomes might explain the impairment of de novo $\mathrm{T}$ cell responses seen in patients with cancer. In line with observations in patients with breast cancer, breast tumour-burdened mice also showed a reduced antigen presentation capacity of dendritic cells. Anti-CD40 treatment, which activated antigen-presenting cells (APCs), induced systemic T cell activity in response to infection. APCs may also be functionally altered in patients with cancer, and this would compromise the immune response. Therefore, combinatorial therapeutic strategies that involve APC activation might be beneficial.

Surgical resection of the tumour tissues restored immune compositional changes in the spleen and the $\mathrm{T}$ cell subsets. The majority of the systemic immune remodelling was also prevented by blocking the tumour-secreted factors interleukin-1 and granulocyte colony-stimulating factor (G-CSF), demonstrating the plasticity of the systemic immune state.

Taken together, this study shows that untreated tumours cause widespread and variable disruption of the immune system over time, both locally and distant from the primary tumour site. These findings provide the foundation for more detailed studies and future clinical trials investigating treatments that re-activate APCs.

Linda Gummlich, Editor, BMC Cancer

ORIGINAL ARTICLE Allen, B. M. et al. Systemic dysfunction and plasticity of the immune macroenvironment in cancer models. Nat. Med. https://doi.org/10.1038/s41591-020-0892-6 (2020) 